\title{
Transcription-driven cohesin repositioning rewires chromatin loops in cellular
}

\author{
senescence
}

loana Olan ${ }^{1}$, Aled J. Parry ${ }^{1,9 *}$, Stefan Schoenfelder ${ }^{2,3 \star}$, Masako Narita ${ }^{1}$, Yoko Ito ${ }^{1}$, Adelyne S.L. Chan $^{1}$, Guy St.C. Slater ${ }^{1}$, Dóra Bihary ${ }^{4}$, Masashige Bando ${ }^{5}$, Katsuhiko Shirahige ${ }^{5}$, Hiroshi Kimura $^{6}$, Shamith A. Samarajiwa ${ }^{4}$, Peter Fraser ${ }^{2,7}$, Masashi Narita ${ }^{1,8}$

${ }^{1}$ Cancer Research UK Cambridge Institute, University of Cambridge, Robinson Way,

10 Cambridge, UK

${ }^{2}$ Nuclear Dynamics Programme, The Babraham Institute, Babraham Research Campus, Cambridge, UK

${ }^{3}$ Epigenetics Programme, The Babraham Institute, Babraham Research Campus, Cambridge, UK

$15{ }^{4}$ MRC Cancer Unit, Hutchison/MRC Research Centre, University of Cambridge, Cambridge Biomedical Campus, Cambridge, UK

${ }^{5}$ Laboratory of Genome Structure and Function, Institute of Molecular and Cellular Biosciences, The University of Tokyo

${ }^{6}$ Cell Biology Centre, Institute of Innovative Research, Tokyo Institute of Technology,

20 Yokohama, Japan

${ }^{7}$ Department of Biological Science, Florida State University, Tallahassee, FL, USA

${ }^{8}$ Tokyo Tech World Research Hub Initiative (WRHI), Institute of Innovative Research, Tokyo Institute of Technology, Yokohama, Japan.

${ }^{9}$ The Babraham Institute, Babraham Research Campus, Cambridge, UK

${ }^{*}$ These authors contributed equally to this work.

Correspondence: M.N. (masashi.narita@cruk.cam.ac.uk) and P.F. (pfraser@bio.fsu.edu) 


\section{Abstract}

Senescence is a phenotypic state of stable proliferative arrest, typically occurring in lineagecommitted cells and triggered by various stimuli. It is generally accompanied by activation of a secretory program (senescence-associated secretory phenotype, SASP), which modulates both local (tissue microenvironment) and systemic (ageing) homeostasis ${ }^{1,2}$. Enhancerpromoter interactions play a key role in gene regulation ${ }^{3-5}$, facilitated by chromatin loops, mostly formed via CCCTC binding factor (CTCF) and cohesin tethering ${ }^{6-8}$. The threedimensional chromatin structure of senescent cells has been characterised ${ }^{9-11}$ mostly in terms of macro-domain structures, but its relevance in gene expression remains elusive. Here, we use $\mathrm{Hi}-\mathrm{C}$ and capture $\mathrm{Hi}-\mathrm{C}^{12,13}$ to show that oncogenic HRAS-induced senescence (RIS) in human diploid fibroblasts (HDFs) is accompanied by extensive enhancer-promoter rewiring, which is closely connected with dynamic cohesin binding to the genome. We find de novo cohesin peaks at the 3' end of a subset of active genes, reminiscent of the transcription-driven 'cohesin islands' recently discovered in mouse embryonic fibroblasts deficient in both CTCF and the cohesin release factor Wings apart-like (Wapl) ${ }^{14}$. RIS de novo cohesin peaks are also transcription-dependent and enriched for SASP genes, as exemplified by $I L 1 B$, where de novo cohesin binding is involved in new loop formation. Cytokine induction is associated with similar cohesin islands appearance and enhancerpromoter rewiring during the terminal differentiation of monocytes to macrophages ${ }^{15}$, but not upon acute TNF $\alpha$ treatment of HDFs ${ }^{16}$. These results suggest that RIS represents a fatedetermined process in which gene expression is regulated beyond the cell type specific 3D chromatin framework, in part through cohesin redistribution. 


\section{Main}

60 Oncogene-induced senescence (OIS) has been linked to dynamic alterations of the chromatin landscape, through the formation of three-dimensional heterochromatic foci ${ }^{17}$, altered distributions of histone modifications and chromatin accessibility ${ }^{2,18}$ or the appearance of

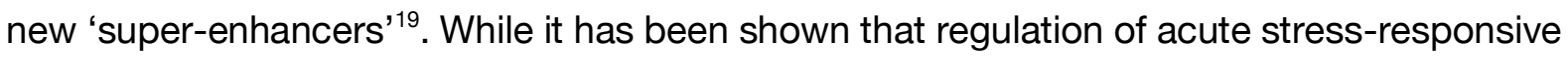
genes can be achieved through transcription factor (TF) recruitment to largely pre-existing enhancer-promoter (EP) contacts ${ }^{16,20,21}$, whether or not a similar mechanism is employed during OIS (sustained oncogenic stress) is unknown. To study gene regulatory mechanisms in the 3D chromatin context at high resolution, we performed in situ $\mathrm{Hi}-\mathrm{C}$ experiments as well as capture $\mathrm{Hi}-\mathrm{C}(\mathrm{cHi}-\mathrm{C})$ for 62 selected genomic regions of interest (Supplementary Table 1) in normal growing and oncogenic HRAS-G12V-induced senescent (RIS) IMR90 human diploid fibroblasts (HDFs), using the 4-hydroxytamoxifen (4OHT)-inducible estrogen receptor (ER) HRAS fusion system ${ }^{22}$ (ER:HRAS ${ }^{\text {G12V }}$ Fig. 1a). Growing (three replicates) and RIS (two replicates) $\mathrm{Hi}-\mathrm{C}$ libraries yielded a total of 523 and 286 million valid reads respectively, after removal of artefacts and duplicates (Supplementary Table 2). There was good agreement between biological replicates, as determined with $\mathrm{HiC}-$ Spector $^{23}$ and by PCA on filtered reads

75 (cHi-C libraries were generated using DNA from the first two growing and RIS Hi-C replicates, Extended Data Fig. 1a-c).

Using these Hi-C data, we identified 3,488 and 3,535 TADs in growing and RIS conditions, respectively. In agreement with a previous study ${ }^{9}$, TAD borders were similar between 80 conditions ( $98 \%$ matched). We found virtually no differences in the distribution of $A / B$ compartments (Extended Data Fig. 2). We then estimated differential interactions between conditions using diffHic ${ }^{24}$ and found extensive alterations in chromatin contacts during RIS within TADs and between distal TADs (Fig. 1b,c), similar to the previous OIS study9. Interestingly, the most extensive change occurred at the location of the NRG1 gene (Fig. 1d), which was strongly up-regulated during RIS. NRG1 was reported as a senescence marker ${ }^{25}$. 
The NRG1 gene was largely (except for a few isoform-specific 5' exons) encompassed in a H3K27me3-dense TAD in growing cells. However, the interactions within the gene body and with the nearby regions were almost entirely lost in RIS cells, thus releasing it from the heterochromatic TAD (Fig. 1e - TADbit modelling, see Methods). This was accompanied by 90 an increase in chromatin accessibility across the gene, as determined by ATAC-seq (Fig. 1d). Similar behaviour was observed in the case of the HMGA2 gene (Extended Data Fig. 1d,e), encoding a regulator of senescence-associated heterochromatic foci ${ }^{26}$ (SAHFs). Genomewide, we identified 102 up-regulated genes dissociating from H3K27me3 regions in RIS (Supplementary Table 3). These data suggest that H3K27me3 regions might contribute to long-range silencing of neighbouring genes through 3D positioning within TADs and that release from such domains appears to be a relatively common mechanism of gene activation during RIS.

We next focused on gene expression and its association with regulatory elements. We 100 annotated differential interactions with active enhancers and promoters (Extended Data Fig. 3) of genes differentially expressed during $\mathrm{RIS}^{27}$. We first used the high resolution ('HindIII resolution', median $4 \mathrm{~kb}$ ) cHi-C data to identify any differential EP pairs. Within the captured regions (Supplementary Table 1), we identified 870 EP pairs that showed significantly altered interactions during RIS, involving 149 differentially expressed genes (Extended Data Fig. 4a).

To gain a genome-wide picture, we next analysed $\mathrm{Hi}-\mathrm{C}$ data and identified 15,618 'EP interactions', which significantly changed at $100 \mathrm{~kb}$ resolution. However, these EP contacts are likely to contain many false positives due to the large bin sizes compared to average enhancer or promoter size. To increase the accuracy of this estimate, we developed a strategy to filter the Hi-C EP interactions by minimising the EP changes annotated in $\mathrm{Hi}-\mathrm{C}$ 
and not in cHi-C over captured regions (likely to be false positives), while maximising the EP changes annotated both in $\mathrm{Hi}-\mathrm{C}$ and $\mathrm{cHi}-\mathrm{C}$ (Extended Data Fig. 4b): enhancers with sizes greater than $7.5 \mathrm{~kb}$ and bin sizes smaller than $30 \mathrm{~kb}$ fulfilled these conditions. Using these filters, we identified 719 EP changes genome-wide from Hi-C data, involving 553 differentially expressed genes (Extended Data Fig. 5a). Combining Hi-C and cHi-C analyses, we identified 1,004 confident EP differential interactions in total (Supplementary Table 2).

The distances between interacting enhancers and promoters from both $\mathrm{Hi}-\mathrm{C}$ and $\mathrm{cHi}-\mathrm{C}$ were below $2 \mathrm{Mb}$, consistent with previous studies $^{16}$. The EP network determined using $\mathrm{cHi}-\mathrm{C}$ 120 showed structures with a wide range of complexity, likely due to the high-resolution interaction information, consisting of 79 components with up to 15 nodes (enhancers or promoters). The complex rewiring was exemplified by the IL 1 and MMP loci, which include major SASP genes (Fig. 2a, Extended Data Fig. 4a). Although the Hi-C EP network, consisting of 479 components, was more disconnected and mostly represented a single EP interaction, the largest component consisted of 13 enhancers differentially interacting with the INHBA gene promoter. Of note, INHBA encodes a SASP factor which has been previously linked to super-enhancer activation in RIS cells ${ }^{19}$. Gene set enrichment analysis using genes involved differential EP interactions (Extended Data Fig. 5b) showed that transcriptionally up-regulated genes (in RIS) were significantly enriched for 'inflammatory' terms, whereas the downregulated genes were enriched for 'cell cycle' terms. This suggests that the two senescence hallmarks, the SASP and proliferative arrest, are controlled through the rewiring of the EP network.

The 'IL1 cluster', which was captured in our cHi-C libraries, encompasses the IL1 ancestral 135 family ${ }^{28}$ (including $I L 1 A, I L 1 B$ ) and several other genes (such as CKAP2L) on chr2q13. Both 
IL1A and IL1B encode key proximal SASP components, which are integral parts of SASP regulation ${ }^{29,30}$. The localization of CKAP2L (encoding a mitotic spindle protein) within the IL1 cluster is highly conserved and the expression of $C K A P 2 L$ is tightly controlled during the cell cycle $^{31}$. Our cHi-C showed dynamic sharing of enhancers between IL1A, IL1B, and CKAP2L

140 during RIS. The differential interaction matrix of $\mathrm{cHi}-\mathrm{C}$ at the IL1 locus showed new loop formation, segregating $I L 1 A$ and $C K A P 2 L$ from $I L 1 B$ and therefore increasing the specificity of their enhancer-associations. Consistently, IL1A and IL1B began to interact more frequently with enhancers located within their respective new loops in RIS (Fig. 2f). Moreover, CKAP2L, which was down-regulated during RIS, interacted less frequently with the same downstream

145 enhancers that $I L 1 B$ began to contact more frequently (Fig. $2 f)$. The data indicate that increased new loop formation and segregation of EP interactions occur at this locus, suggesting new loop formation around the IL1B gene.

150 This finding is in marked contrast to the IL1 induction in a TNF $\alpha$ acute inflammatory scenario, in which gene regulation can be achieved without any detectable alteration to the EP landscape. Using high-resolution $(5-10 \mathrm{~kb}) \mathrm{Hi}-\mathrm{C}$ maps, Jin et al. ${ }^{16}$ have shown that a transient TNF $\alpha$ treatment of IMR90 cells leads to upregulation of IL1A and IL1B with increased binding of NF-kB (a major inflammatory TF) to active enhancers of its targets. In addition, IL1A and CKAP2L were shown to be induced simultaneously via shared enhancer binding. The authors concluded that gene expression alterations mostly occur via TF binding to 'pre-existing' EP complexes, at least upon $\mathrm{TNF} \alpha$ treatment $^{16}$. We reanalysed the $\mathrm{Hi}-\mathrm{C}$ data from this study using the presently described method and, like Jin et al., did not observe any significant changes upon TNF $\alpha$ treatment (Fig. 2c,e). This reveals a fundamentally distinct mechanism 160 for the induction of inflammatory cytokines during senescence and acute inflammation. The anti-correlation between $I L 1$ and CKAP2L expression with significant EP interaction 
alterations was observed during RIS, but not with TNF $\alpha$ treatment, implying a senescencespecific decoupling mechanism within an otherwise co-regulated locus encoding key cytokines and cell cycle genes.

To investigate potential mechanisms underlying the observed EP changes during RIS, we generated ChIP-seq data for CTCF and cohesin (RAD21 and SMC3), chromatin structural proteins associated with chromatin loops ${ }^{6,32}$, in both growing and RIS IMR90 cells. We found 44,764 and 53,563 CTCF peaks in growing and RIS cells, respectively. Comparative analysis identified 1,774 CTCF peaks that were significantly altered during RIS. $96 \%$ of the CTCF changes were associated with increased binding in RIS (Fig. 3a). In contrast, RAD21 binding, represented by 26,374 and 24,355 peaks in growing and RIS, respectively, changed significantly at 4,553 sites, of which $81 \%$ corresponded to decreased binding (Fig. 3a).

175 Similar results were obtained for SMC3 ChIP-seq which correlates well with RAD21 ChIPseq signal (Fig. 3d-f, genome-wide Pearson correlation values between 0.73 and 0.96 ). Thus, although substantial numbers of peaks were gained in both CTCF and cohesin, a large fraction of cohesin binding was diminished.

180 Next, we investigated where the CTCF and cohesin binding changes occurred with regards to genomic features and loops (the latter previously defined in normal IMR90 cells by Rao et al. ${ }^{6}$ (Fig. 3b, see Methods). 3,407 (out of 7,647) loops showed changes in cohesin binding, mostly decreases (80\%), at their anchors, whereas only 363 showed any CTCF changes. Such strong colocalization between cohesin loss and loop anchors suggests that extensive 185 loop reorganization might occur during RIS, mostly through redistribution of cohesin rather than CTCF (Fig. 3b). To visualize the relationship between cohesin reduction at loop ends and their physical contacts, we aggregated interaction neighbourhoods (at $20 \mathrm{~kb}$ resolution) 
centred on selected loops (Fig. 3c, Methods), a similar approach to the previously published method 'Aggregate Peak Analysis' ${ }^{6}$. Loops with cohesin loss at one or both ends $(1,827$

190 loops) showed a trend of decreased interaction in RIS compared to growing cells (Fig. 3c, left). More stringently, 326 loops (Fig. 3c, right) were found to overlap with significantly reduced interactions during $\mathrm{RIS}$, either from $\mathrm{cHi}-\mathrm{C}$ or $\mathrm{Hi}-\mathrm{C}$ (at $20 \mathrm{~kb}$ and $40 \mathrm{~kb}$ resolution). In terms of enhancer-promoter interactions affected, 200 differential EP contacts were nested within those robustly diminished loops, involving 92 genes, including the cell cycle regulator

195 CCNA2 (Extended Data Fig. 6).

Interestingly, the vast majority of cohesin binding increases occurred de novo in RIS (Extended Data Fig. 7), compared to the decreased binding which did not result in complete binding loss. The genes and enhancers studied in the IL1 locus belong to a single loop 200 identified in IMR90 cells ${ }^{6}$. This is consistent with enhancer sharing between these genes and their co-regulation in response to TNF $\alpha$ in these cells ${ }^{16}$. We found a de novo cohesin peak close to the 3' end of IL1B in RIS cells, independent of CTCF binding (Fig. 3d). A similar cohesin peak was observed when RIS was induced via constitutive expression of HRAS ${ }^{\mathrm{G} 12 \mathrm{~V}}$ without the ER-tag not only in IMR90, but also in WI38 HDFs (Fig. 3e). These data suggest

205 that loop reorganization at the IL 1 locus is associated with de novo cohesin binding (Fig. 2df). Importantly, increased cohesin occupancy or altered regulatory chromatin interactions at the 3' end of $I L 1 B$ were not observed in response to TNF $\alpha$ treatment, where no new loops were detected (Fig. 2e,f, Fig. 3f). Additionally, we observed an increase in the contact intensity between the new cohesin peak and the anchors of the loop (Fig. 2d,f). Collectively,

210 these data suggest that the de novo cohesin peak contributes to the formation of new loops in the IL1 locus and that within each loop domain, EP pairs might preferentially contact (Fig. 3g). Strikingly, the MMP locus, which contains other major SASP genes, was also characterized by the appearance of de novo cohesin at the 3' end of MMP1 (and, to a lesser 
extent, MMP3), as well as loop reorganization around the new cohesin peak (Extended Data

215 Fig. 8a,b). We confirmed that the cohesin increases also occurred in RIS WI38 cells (Extended Data Fig. 8c).

The elongated shape of the cohesin peaks at the 3' end of IL1B and MMP1 was reminiscent of recently reported ${ }^{14}$ transcription-driven 'cohesin islands', which appear at the 3 ' end of active convergent genes in double knockout (DKO) mouse embryonic fibroblasts (MEFs) of Ctcf and Wapl (the cohesin releasing factor). The authors proposed that cohesin is loaded onto chromatin at the TSSs of a large number of active genes and is then relocated though transcription: if there is no CTCF in the way and no efficient cohesin release at the 3 ' end of active genes, cohesin accumulates at the $3^{\prime}$ end of these genes ${ }^{14}$. A similar pattern of cohesin 225 binding has been reported in wild-type yeast, which lacks a CTCF equivalent ${ }^{33-35}$. Thus, we hypothesized that genes highly active in RIS somehow allow for the accumulation of cohesin at their 3' ends in a transcription-dependent manner, potentially promoting loop reorganization. To test this, we compared transcript abundance and cohesin binding at the 3' end of genes. Both convergent (regions downstream their 3' ends overlap) and isolated 230 (no overlap with other genes) genes in RIS IMR90 cells accumulated cohesin islands and cohesin binding correlated with gene expression (Fig. 4a, Extended Data Fig. 9a,b). Consistent with the lack of cohesin islands detected in wild-type MEFs by Busslinger et al. ${ }^{14}$, very few cohesin islands were detected in normal growing IMR90 cells. To confirm that cohesin islands were associated with the cellular condition, rather than a specific subset of active genes, we examined genes highly transcribed in both RIS and growing, but at higher levels in growing cells, for cohesin islands. Despite the reduced expression levels, cohesin islands were much more pronounced in the RIS condition (Extended Data Fig. 9c). 
240 To further investigate the transcriptional dependence of cohesin islands observed in RIS cells, we performed ChIP-seq experiments in RIS cells with or without 5,6-dichloro-1- $\beta$-D-ribofuranosylbenzimidazole (DRB) treatment, a transcription elongation inhibitor. Strikingly, the de novo cohesin peaks at the $I L 1 B$, as well as MMP1 sites, disappeared completely (Fig. 4b and Extended Data Fig. 8d). We used this information to

245 define genome-wide RIS-associated cohesin islands. We found 574 wide cohesin peaks (between 2 and $20 \mathrm{~kb}$ wide), which were lost with DRB treatment (Fig. 4c). These regions overlapped 343 highly expressed genes in RIS, near their 3' ends (Supplementary Table 3). In addition to IL1B and MMP1 (discussed above), these genes were enriched in pathways that have been implicated in senescence, such as Wnt, Autophagy and NF- $\kappa$ B signalling ${ }^{2,36,37}$

250 (Extended Data Fig. 9d), and included key SASP factors. Interestingly, cohesin islands in RIS cells appeared to lack CTCF binding (Fig. 4c), suggesting that, similar to the cohesin islands defined in knockout MEFs ${ }^{14}$, their formation is CTCF-independent. The same cohesin islands also occurred in RIS IMR90 and WI38 cells, but not in TNF $\alpha$-treated IMR90 cells (Extended Data Fig. 9e,f).

Next, we assessed whether RIS-associated cohesin islands modulate local chromatin structure, as observed at the IL 1 and MMP loci. Interactions between all cohesin islands and surrounding cohesin peaks (2,512 interactions) were increased in RIS (Fig. 4d, left). 105

260 cohesin islands exhibited significantly increased binding to local cohesin peaks (259 interactions, Fig. 4d, right). These data suggest that cohesin islands likely contribute to changes in chromatin architecture during RIS, via de novo loop formation.

265 Finally, we asked whether changes similar to those observed at the IL1 locus in RIS cells occur in any other context. In most other cell types with cohesin information from ENCODE, 
the IL1 locus cohesin binding pattern was similar to 'normal' IMR90 cells, suggesting that the loop structure at this locus is mostly conserved. However, Phanstiel et al. ${ }^{15}$ recently reported IL1B up-regulation and concomitant loop formation at the locus during terminal differentiation of monocytes into macrophages, another fate-determination process of lineage committed cells. They generated high-resolution Hi-C maps and RNA-seq datasets in the monocytic leukaemia cell line THP-1 both before (monocytes) and after (macrophages) PMA treatment, which we reanalysed. Strikingly, new loop formation in the IL1 locus in THP-1 macrophages was similar to RIS (Fig. 4e), concomitant with similar expression changes: up-regulation of

275 IL1A and IL1B and down-regulation of CKAP2L. Reanalysing RAD21 ChIP-seq data in the same THP-1 cell model from Heinz et al. ${ }^{38}$ revealed a de novo cohesin peak around IL1B (Fig. 4e). Genome-wide, cohesin binding also correlated with transcription levels (Extended Data Fig. 9g). Interestingly, 65 genes exhibited cohesin islands in both RIS and THP-1 macrophages (Extended Data Fig. 9h). Together, these data suggest that transcriptiondependent cohesin accumulation also occurs during macrophage terminal differentiation and particularly, the same cohesin-mediated loop alteration at the IL1 locus (as in RIS) facilitate transcription of genes in this locus.

We show that significantly altered EP contacts, associated with gene expression changes, occur during RIS. This is in stark contrast to proinflammatory gene expression programs in response to acute stress or signalling cues, which appear to be predominantly driven by TF recruitment and remodelling of epigenetic chromatin signatures, rather than by dynamic alteration of EP interactions. Our data indicate that EP contacts in HDFs exhibit plasticity, being susceptible to further modulation towards senescence. EP contacts in lineagecommitted cells also exhibit plasticity towards terminal differentiation ${ }^{15,21}$. Mechanistically, our data suggest that this can be at least in part explained by the formation of transcriptiondependent cohesin islands. Indeed, we also observed the induction of cohesin islands during 
macrophage differentiation (Fig. 4e). Generally, cohesin islands tend to be longer than the CTCF-associated structural cohesin peaks, but shorter in RIS HDFs and macrophages (up to $20 \mathrm{~kb}$ ) than in Ctcf/Wapl DKO MEFs ${ }^{14}$ (up to $70 \mathrm{~kb}$ ). Although the trigger is unclear, it is tempting to speculate that the initial de novo cohesin accumulation promotes new loop formation, and thus, increased gene expression. This would further promote transcriptiondependent cohesin accumulation, constituting a gene amplification feed-forward mechanism. Our data highlight that such accumulation of cohesin islands does occur in physiological contexts in mammalian cells, where they potentially constitute an additional layer of gene regulation for cell fate determination, by modulating higher-order chromatin structure.

1. Coppé, J.-P., Desprez, P.-Y., Krtolica, A. \& Campisi, J. The Senescence-Associated Secretory Phenotype: The Dark Side of Tumor Suppression. Annu. Rev. Pathol. Mech. Dis. 5, 99-118 (2010).

2. Chan, A. S. L. \& Narita, M. Short-term gain, long-term pain: the senescence life cycle and cancer. Genes Dev. 33, 127-143 (2019).

3. Javierre, B. M. et al. Lineage-Specific Genome Architecture Links Enhancers and $310 \quad$ Non-coding Disease Variants to Target Gene Promoters. Cell 167, 1369-1384.e19 (2016).

4. Bonev Boyan, Cavalli Giacomo, Bonev, B. \& Cavalli, G. Organization and function of the 3D genome. Nat. Rev. Genet. 17, 661-678 (2016).

5. Schoenfelder, S. \& Fraser, P. Long-range enhancer-promoter contacts in gene expression control. Nat. Rev. Genet. 20, 437-455 (2019).

6. Rao, S. S. P. S. P. et al. A 3D map of the human genome at kilobase resolution reveals principles of chromatin looping. Cell. 159, 1665-1680 (2014).

7. Merkenschlager, M. \& Nora, E. P. CTCF and Cohesin in Genome Folding and 
Transcriptional Gene Regulation. Annu. Rev. Genomics Hum. Genet. 17, 17-43

320 (2016).

8. Rowley, M. J. \& Corces, V. G. Organizational principles of 3D genome architecture. Nat. Rev. Genet. 19, 789-800 (2018).

9. Chandra, T. et al. Global Reorganization of the Nuclear Landscape in Senescent Cells. Cell Rep. 10, 471-483 (2015).

325 10. Criscione, S. W. et al. Reorganization of chromosome architecture in replicative cellular senescence. Sci. Adv. 2, e1500882 (2016).

11. Zirkel, A. et al. HMGB2 Loss upon Senescence Entry Disrupts Genomic Organization and Induces CTCF Clustering across Cell Types. Mol. Cell 70, 730-744.e6 (2018).

12. Mifsud, B. et al. Mapping long-range promoter contacts in human cells with highresolution capture Hi-C. Nat. Genet. 47, 598-606 (2015).

13. Schoenfelder, S. et al. The pluripotent regulatory circuitry connecting promoters to their long-range interacting elements. Genome Res. 25, 582-97 (2015).

14. Busslinger, G. A. et al. Cohesin is positioned in mammalian genomes by transcription, CTCF and Wapl. Nature 544, 503-507 (2017).

335 15. Phanstiel, D. H. et al. Static and Dynamic DNA Loops form AP-1-Bound Activation Hubs during Macrophage Development. Mol. Cell 67, 1037-1048.e6 (2017).

16. Jin, F. et al. A high-resolution map of the three-dimensional chromatin interactome in human cells. Nature 503, 290-294 (2013).

17. Narita, M. M. et al. Rb-Mediated Heterochromatin Formation and Silencing of E2F Target Genes during Cellular Senescence. Cell 113, 703-716 (2003).

18. Rai, T. S. \& Adams, P. D. Lessons from senescence: Chromatin maintenance in nonproliferating cells. Biochim. Biophys. Acta - Gene Regul. Mech. 1819, 322-331 (2012).

19. Tasdemir, N. et al. BRD4 Connects Enhancer Remodeling to Senescence Immune 
Surveillance. Cancer Discov. 6, 612-29 (2016).

20. Kolovos, P. et al. Binding of nuclear factor $\mathrm{kB}$ to noncanonical consensus sites reveals its multimodal role during the early inflammatory response. Genome Res. 26, 1478-1489 (2016).

21. Comoglio, F. et al. Thrombopoietin signaling to chromatin elicits rapid and pervasive epigenome remodeling within poised chromatin architectures. Genome Res. (2018). doi:10.1101/gr.227272.117

22. Young, A. R. J. J. et al. Autophagy mediates the mitotic senescence transition. Genes Dev. 23, 798 (2009).

23. Yan, K.-K. et al. HiC-spector: a matrix library for spectral and reproducibility analysis of Hi-C contact maps. Bioinformatics 33, 2199-2201 (2017).

24. Lun, A. T. L. L. \& Smyth, G. K. diffHic: a Bioconductor package to detect differential genomic interactions in Hi-C data. BMC Bioinformatics 16, 258 (2015).

25. Baker, D. J. et al. Opposing roles for p16lnk4a and p19Arf in senescence and ageing caused by BubR1 insufficiency. Nat. Cell Biol. 10, 825-36 (2008).

360 26. Narita, M. M. et al. A Novel Role for High-Mobility Group A Proteins in Cellular Senescence and Heterochromatin Formation. Cell 126, 503-514 (2006).

27. Hoare, M. et al. NOTCH1 mediates a switch between two distinct secretomes during senescence. Nat. Cell Biol. 18, 979-992 (2016).

28. Rivers-Auty, J., Daniels, M. J. D., Colliver, I., Robertson, D. L. \& Brough, D. Redefining the ancestral origins of the interleukin-1 superfamily. Nat. Commun. 9, 1156 (2018).

29. Orjalo, A. V., Bhaumik, D., Gengler, B. K., Scott, G. K. \& Campisi, J. Cell surfacebound IL-1 is an upstream regulator of the senescence-associated IL-6/IL-8 cytokine network. Proc. Natl. Acad. Sci. 106, 17031-17036 (2009).

370 30. Acosta, J. C. et al. A complex secretory program orchestrated by the inflammasome 
controls paracrine senescence. Nat. Cell Biol. 15, 978-990 (2013).

31. Yumoto, T. et al. Radmis, a Novel Mitotic Spindle Protein that Functions in Cell Division of Neural Progenitors. PLoS One 8, e79895 (2013).

32. Heidari, N. et al. Genome-wide map of regulatory interactions in the human genome.

Genome Res. 24, 1905-17 (2014).

33. Glynn, E. F. et al. Genome-Wide Mapping of the Cohesin Complex in the Yeast Saccharomyces cerevisiae. PLoS Biol. 2, e259 (2004).

34. Gullerova, M. \& Proudfoot, N. J. Cohesin Complex Promotes Transcriptional Termination between Convergent Genes in S. pombe. Cell 132, 983-995 (2008).

35. Lengronne, A. et al. Cohesin relocation from sites of chromosomal loading to places of convergent transcription. Nature 430, 573-8 (2004).

36. Ye, X. et al. Downregulation of Wnt Signaling Is a Trigger for Formation of Facultative Heterochromatin and Onset of Cell Senescence in Primary Human Cells. Mol. Cell 27, 183-196 (2007).

385 37. Kuilman, T., Michaloglou, C., Mooi, W. J. \& Peeper, D. S. The essence of senescence. Genes Dev. 24, 2463-2479 (2010).

38. Heinz, S. et al. Transcription Elongation Can Affect Genome 3D Structure. Cell 174, 1522-1536.e22 (2018).

\section{Methods}

Cell culture. IMR90 and WI38 HDFs (ATCC) were cultured in Dulbecco's modified Eagle's medium (DMEM)/10\% foetal calf serum (FCS) in a 5\% O2/5\% CO2 atmosphere. Cell identity was confirmed by STR (short tandem repeats) genotyping. Cells were regularly tested for 395 mycoplasma contamination and always found to be negative. The following compounds were used in cultures: $100 \mathrm{nM}$ 4-hydroxytamoxifen (4OHT) (Sigma, cat\#H7904), $100 \mu \mathrm{M}$ 5,6- 
dichloro-1- $\beta$-D-ribofuranosylbenzimidazole (DRB) (Sigma, cat\#D1916), $10 \mathrm{ng} / \mathrm{mL}$ tumour necrosis factor alpha (TNFa) (PeproTech, cat\#300-01A) as indicated in individual figures.

Vectors. The following retroviral vectors were used: pLNCX2 (clontech) for ER:HRAS ${ }^{\mathrm{G} 12 \mathrm{~V}}$ 400 (Young et al. ${ }^{22}$ ), pBabe-puro for HRas ${ }^{\mathrm{G} 12 \mathrm{~V}}$. Senescence was induced using the ER:RAS system unless otherwise mentioned.

ChIP-seq. Chromatin immunoprecipitation (ChIP) was performed as previously described ${ }^{39,40}$ for the following antibodies: anti-H3K27ac ${ }^{41}$ (clone CMA309), anti-H3K27me3 ${ }^{41}$ (clone CMA323), anti-CTCF (Cell Signaling Technology, clone D31H12, \#3418), anti-RAD21 ${ }^{42}$ and 405 anti-SMC3 (Abcam ab9263). Libraries were prepared using the NEBNext Ultra II DNA Library Prep Kit for Illumina (New England Biolabs, cat\#E7645L) according to the manufacturer's instructions except that size selection was performed after PCR amplification using AMPure XP beads (Beckman Coulter, cat\#A63881). Samples were sequenced paired-end using 50 bp reads on the Illumina platforms.

$410 \mathrm{Hi}-\mathrm{C}$ and capture $\mathrm{Hi}-\mathrm{C}$. $\mathrm{Hi}-\mathrm{C}$ and capture $\mathrm{Hi}-\mathrm{C}$ libraries were generated as previously described $^{12,13,43}$ using the in-nucleus ligation protocol ${ }^{44}$. For each sample and replicate 50 million IMR90 cells were used. For capture Hi-C, biotinylated 120-mer RNA baits complementary to both ends of each target HindIII restriction fragment of interest were designed. Target sequences were valid if they contained no more than two consecutive N's, 415 were within 330bp of the HindIII restriction site and had a GC content ranging between 25 and $65 \%$.

Hi-C data processing. $\mathrm{Hi}-\mathrm{C}$ and $\mathrm{CHi}-\mathrm{C}$ libraries were aligned with $\mathrm{HiC}-\mathrm{Pro}^{45}$, against the hg19 genome build. Artefacts were identified and removed using both $\mathrm{HiC}$-Pro and diffHic ${ }^{24}$ ( $\mathrm{R}$ Bioconductor package) and reads were counted into bins at several resolutions (HindIII and $5 \mathrm{~kb}$ for $\mathrm{cHi}-\mathrm{C}$ and $10 \mathrm{~kb}-100 \mathrm{~kb}$ for $\mathrm{Hi}-\mathrm{C})$. Read duplicates were removed using samtools ${ }^{46}$ markdup. We used $\mathrm{HiC}-$ Spector $^{23}$ to check for the similarity between biological 
replicates, as well as PCA on library size normalized interaction matrices which were filtered for low counts and diagonal entries, produced with diffHic.

A/B compartments. A/B compartments were called as before ${ }^{47}$, by performing PCA on 425 distance-corrected, ICE-normalized $\mathrm{Hi}-\mathrm{C}$ matrices at $100 \mathrm{~kb}$ resolution. The principal component which correlated well in absolute value with H3K4me1 ChIP-seq signal was chosen as representative of $A / B$ compartments. The sign of the $A / B$ compartments vector was set to match the sign of the correlation with H3K4me1 signal so that A compartment regions were represented by positive values and $\mathrm{B}$ compartment regions were represented 430 by negative values.

TADs. TADs were called using TADbit ${ }^{48}$ from $\mathrm{Hi}-\mathrm{C}$ matrices at $40 \mathrm{~kb}$ resolution. A confidence score between 1 and 10 was assigned to each TAD border by TADbit. TADs from biological replicates were combined in a consensus set per condition using TADbit and only considering TAD borders with scores over 7 (out of 10).

435 Interaction Modelling. We used TADbit ${ }^{48}$ to compute 3D models of the interactions of genes released from $\mathrm{H} 3 \mathrm{~K} 27 \mathrm{me} 3$ neighbourhoods using the ICE-normalized matrices at $20 \mathrm{~kb}$ resolution, combined across biological replicates for growing and RIS, respectively. The matrices used correspond to the subset of interactions of one of two TADs around each gene of interest. In each case, we tried several parameter spaces for IMP parameter optimisation, 440 employed by TADbit. For each region, we then chose the parameter subspace which fit the interaction values curve best. Modelling with $\mathrm{IMP}^{49}$ within TADbit was then performed with the parameters optimised for each case. The top 10 models predicted in each case were selected and exported from TADbit as XYZ coordinates.

Differential interaction analysis. We performed differential interaction analysis between 445 growing and $\mathrm{RIS} \mathrm{Hi-C} \mathrm{and} \mathrm{cHi}-\mathrm{C}$ libraries at several resolutions (HindIII and $5 \mathrm{~kb}$ for $\mathrm{cHi}-\mathrm{C}$ and $10 \mathrm{~kb}-100 \mathrm{~kb}$ for $\mathrm{Hi}-\mathrm{C}$, increasing in $5 \mathrm{~kb}$ steps) using diffHic ${ }^{24}$ (R Bioconductor). 
Libraries with artefacts and duplicates removed were further filtered for low counts and diagonal entries. Using diffHic, we performed non-linear normalization (LOESS) to remove trended biases between libraries. We tested for significant interaction changes at 5\% FDR by using quasi-likelihood F-tests and Benjamini-Hochberg multiple testing correction from diffHic.

Enhancer-promoter interactions were annotated by checking the bins involved in significant differential interactions for overlaps with enhancers and promoters. We used cHi-C EP interactions annotated using HindIIl fragments and we combined Hi-C EP interactions determined at $10 \mathrm{~kb}, 15 \mathrm{~kb}, 20 \mathrm{~kb}, 25 \mathrm{~kb}$ and $30 \mathrm{~kb}$, filtered for enhancers longer than $7.5 \mathrm{~kb}$. Enhancers were determined as before ${ }^{19}$, using H3K27ac peaks which overlap ATAC-seq peaks in each condition and collapsing peaks nearer than $12 \mathrm{~kb}$. Promoters were represented as $5 \mathrm{~kb}$ regions around the TSS of protein-coding genes (GENCODE v19 reference). Only promoters of differentially expressed genes in RIS were considered.

460 Using $\mathrm{cHi}-\mathrm{C}$ to filter EP interactions determined with $\mathrm{Hi}-\mathrm{C}$. In order to annotate EP interactions from $\mathrm{Hi}-\mathrm{C}$ more robustly, we compared several filtering strategies at different resolutions, using the contacts detected using $\mathrm{cHi}-\mathrm{C}$ as a baseline for comparison, due to their accuracy at high resolution (HindIII). We wanted to maximise the number of EP interactions detected in the captured regions from both $\mathrm{Hi}-\mathrm{C}$ and $\mathrm{cHi}-\mathrm{C}$ and to minimise the 465 interactions detected from $\mathrm{Hi}-\mathrm{C}$ but not from $\mathrm{cHi}-\mathrm{C}$, which were likely false positives. We tried selecting only interactions involving enhancers of large sizes (over $5 \mathrm{~kb}, 7.5 \mathrm{~kb}$ or $10 \mathrm{~kb}$ ) or genes which were more robustly differentially expressed in RIS (FDR $<0.01$ ), as well as selecting bins without other regulatory elements. All of these filters were applied on EP interactions detected at resolutions between $10 \mathrm{~kb}$ and $100 \mathrm{~kb}$ as bin size can also affect the 470 accuracy of the interactions detected. Finally, we selected resolutions higher than $30 \mathrm{~kb}$ (10 $\mathrm{kb}, 15 \mathrm{~kb}, 20 \mathrm{~kb}, 25 \mathrm{~kb}, 30 \mathrm{~kb}$ ) and interactions involving enhancers larger than $7.5 \mathrm{~kb}$ for the EP interactions annotated from $\mathrm{Hi}-\mathrm{C}$. 
ChIP-seq analysis. ChIP-seq libraries were aligned against the hg19 genome build using bowtie $2^{50}$ and uniquely mapping reads which did not bind to 'blacklisted regions' ${ }^{51,52}$ were 475 used for further analysis. Peak calling was performed using MACS2 ${ }^{53}$ with insert sizes calculated using the R Bioconductor ChIPQC package ${ }^{54}$. Consensus peak sets were calculated for each condition by selecting peaks which appear in at least two replicates. Differential binding analysis was performed using $\mathrm{THOR}^{55}$ and a custom R script was used to filter for significant binding changes at 5\% FDR and a minimum of 100 reads per location

480 in at least one of the conditions. DeepTools ${ }^{56}$ was used to calculate and visualize ChIP-seq profiles summarized across genomic regions.

RNA-seq analysis. RNA-seq libraries were aligned using $\operatorname{STAR}^{57}$ against the hg19 genome build and reads were counted against genes (GENCODE v19 reference) using subread ${ }^{58}$. Differential expression analysis was performed using glmTREAT from edge ${ }^{59}$ and 485 significantly differentially expressed genes were selected at 5\% FDR. log-TPM expression values were also calculated for the analysis of transcription and cohesin binding. Gene enrichment analysis of sets of genes of interest was performed using the enrichR $R$ package (CRAN https://cran.r-project.org) which queries EnrichR ${ }^{60,61}$ against the WikiPathways database.

490 Data visualization. Hi-C matrices corresponding to combined biological replicates were used for visualization. The raw matrices for each replicate were combined by calculating the overall negative binomial mean contacts with correctedContact from diffHic ${ }^{24}$ and further normalized using ICE ${ }^{62}$ and distance correction as part of the correctedContact functionality. Plotting the matrices was performed using a custom set of R scripts consisting of horizontal 495 rotation of the matrix coordinates and overlaid genomic information such as enhancer and promoter positions, ChIP-seq tracks or interaction arcs. Interactions were coloured using a non-linear scale which represented interaction values above and below the expected values (positive and negative values, respectively, determined with distance correction) with 'warm' 
and 'cold' colours, respectively. In the case of overlaying cHi-C matrices at HindIII resolution, each interaction unit was represented as equally sized, despite the variable lengths of HindIII fragments. ChIP-seq tracks represented were THOR-normalized (input subtracted and library-normalized) bigWig files produced during the differential binding analysis. The tracks were exported from IGV ${ }^{63}$ and were scaled to be within the same interval, to allow for comparison between conditions. RNA-seq bigWig files were also produced for visualization

505 of expression and TMM factors were used for normalization of the signal between libraries. Models derived from $\mathrm{Hi}-\mathrm{C}$ interactions using TADbit ${ }^{48}$ were visualized using the $\mathrm{R}$ package rgl (CRAN https://cran.r-project.org). The points corresponding to a model are centred around 0 . The curve used to visualize the model is drawn by adding 10 additional points between every pair of points in the set of original coordinates by interpolation with the spline 510 function in $\mathrm{R}$.

Cohesin Islands. We investigated the association between cohesin accumulation and transcription by grouping genes by expression level (represented as log-TPM averaged across biological replicates) and plotting their SMC3 and RAD21 ChIP-seq profile. The binding profile was centred either at their transcription end site (TES) in the case of isolated

515 genes or in the middle of the genomic region bounded by TES of two convergent genes. We focused this analysis on isolated and convergent genes, like in Busslinger et al. ${ }^{14}$, in order to avoid biases caused by genes with overlapping regions post 3'UTR. Small increases in signal were observed before the TES as well caused by short genes which show cohesin binding on their gene bodies as well.

520 We determined cohesin islands by comparing the RIS cohesin ChIP-seq libraries with and without DRB treatment (transcription elongation inhibitor). We then selected significantly differentially bound regions larger than $2 \mathrm{~kb}$. We eliminated possible false positives which can occur due to genes with overlapping 3' end regions (such as convergent genes) by 
filtering for highly expressed genes. Genes with cohesin islands were determined by overlapping $10 \mathrm{~kb}$ regions starting at the TES with the cohesin islands determined.

Interaction neighbourhood aggregation. We identified general trends of certain subsets of interactions by selecting a two-dimensional neighbourhood around each interaction of interest, and summing the corresponding Hi-C sub-matrix (from the ICE-normalized and distance-corrected matrix averaged across replicates, as described earlier), similarly to

530 Aggregate Peak Analysis ${ }^{6}$. Each interaction pixel was divided by the number of sub-matrices added minus the number of missing values. We selected a $200 \mathrm{~kb}$ region around each bin containing a cohesin peak of interest which, at $20 \mathrm{~kb}$ resolution, resulted in neighbourhoods of $11 \times 11$ pixels. Differential aggregated matrices were computed by subtracting the growingspecific aggregated matrix from the RIS one.

535 Data availability. $\mathrm{Hi}-\mathrm{C}$ and $\mathrm{cHi}-\mathrm{C}$ data in growing and senescent IMR90 cells, as well as ChIP-seq data in IMR90 and WI38 human diploid fibroblasts in the growing (with and without $\mathrm{TNF} \alpha$ treatment) and RIS (with and without DRB treatment) conditions were deposited in the Gene Expression Omnibus: GSExxxxx (will be made public upon publication). Publicly available ChIP-seq data were also reanalysed in this study: RAD21 and CTCF ChIP-seq in 540 monocyte (THP-1) and macrophage (PMA-induced) controls from Heinz et al. ${ }^{38}$ (GSE103477), H3K4me3 and H3K27me3 ChIP-seq from Chandra et al. ${ }^{64}$ (GSE38448), H3K27ac ChIP-seq and ATAC-seq from Parry et al. ${ }^{65}$ (GSE103590). Publicly available RNA-seq data from Hoare et al. ${ }^{27}$ (GSE72404), Phanstiel et al. ${ }^{15}$ (GSE96800) and Jin et al. ${ }^{16}$ (GSE43070) were reanalysed in this study. Publicly available Hi-C data from Phanstiel et al. ${ }^{15}$ (PRJNA385337) and Chandra et al. ${ }^{9}$ (PRJEB8073) were also reanalysed in this study.

Code availability. Custom scripts used for enhancer-promoter annotation and filtering THOR differential binding output were uploaded to the OSF public repository (will be made public 
upon publication). Visualisation scripts for the $\mathrm{Hi}-\mathrm{C}$ matrices are also available on GitLab (https://gitlab.com/ilyco/hicvizr).

\section{Acknowledgements}

We thank all members of the Narita laboratory for helpful discussions, CRUK-Cl core facilities (Genomics, Biorepository, Bioinformatics and Research Instrumentation) for technical support. This work was supported by a Cancer Research UK Cambridge Institute Core Grant (C9545/A29580) to the M.N. laboratory. M.N. was also supported by the Medical Research

555 Council (MR/M013049/1) and BBSRC (BB/S013466/1). I.O. was supported by Wellcome Trust (105367/Z/14/Z). S.S. was supported by the Biotechnology and Biological Science Research Council UK (BB/J004480/1), a Career Progression Fellowship from the Babraham Institute, and an MRC UKRI Rutherford Fund Fellowship. A.J.P. was supported by a Sir Henry Wellcome Postdoctoral Fellowship (215912/Z/19/Z). H.K. was supported by JSPS KAKENHI

560 (JP17H01417 and JP18H05527) and JST-CREST (JPMJCR16G1). K.S. and M.B. were supported by Grant-in-Aid for Scientific Research on Innovative Areas (15H05976). S.A.S and D.B. are supported by the Medical Research Council (MC_UU_12022/10).

\section{Author contributions}

I.O. and M.N. conceived the study. A.J.P., S.S. and $\mathrm{YI}$ performed Hi-C and capture Hi-C experiments. P.F. supervised $\mathrm{Hi}-\mathrm{C} /$ capture $\mathrm{Hi}-\mathrm{C}$ experiments and interpreted the data. Masako N. and A.J.P. performed the ChIP-seq experiments with help by H.K., K.S., and M.B. G.St.C.S. performed three-dimensional interaction modelling and visualization. I.O. analysed the Hi-C, cHi-C, ChIP-seq and RNA-seq data with input from D.B., S.A.S., and A.S.L.C. M.N. and I.O. wrote the manuscript with input from the other authors. 


\section{Competing interests}

The authors declare that they have no competing financial interests.

\section{$575 \quad$ Figure Legends}

Figure 1 Changes in chromatin interactions identified with $\mathrm{Hi}-\mathrm{C}$ during RIS: a, Experimental setup of control Growing and RAS-induced senescent (RIS) IMR90 cells. ROI, regions of interest. b, Number of significant interaction changes during RIS at resolutions between 10 and $100 \mathrm{~kb}$. c, Hi-C matrices (300 kb resolution) of chromosome 8 in Growing and RIS cells; arcs represent significant interaction changes (100 kb resolution); black boxes represent captured regions. d, Hi-C interaction matrices at the NRG1 locus, marked by dotted lines in $\mathbf{c}$ at $20 \mathrm{~kb}$ resolution, with matching tracks for RNA-seq, ChIP-seq, and ATACseq; coloured triangles represent TADs. e, Three-dimensional interaction modelling with TADbit at the NRG1 locus in Growing and RIS, including NRG1 (green) and surrounding TADs marked in $\mathbf{d}$.

Figure 2 Reorganization of the local chromatin neighbourhood at the IL1 locus: a, Differential enhancer-promoter contacts involving $I L 1$ and $M M P$ in $\mathrm{cHi}-\mathrm{C}$. $\mathbf{b}-\mathbf{c}, \mathrm{Hi}-\mathrm{C}$ matrices (10 kb resolution) of the IL1 locus corresponding to growing and RIS IMR90 cells (b) and control and TNF $\alpha$-treated IMR90 cells from Jin et al. ${ }^{16}$ (c). d, Differential capture Hi-C matrix

590 at the IL1 locus (log-fold change of RIS/growing interactions) at HindIII resolution, with annotated growing IMR90 loops (from Rao et al. ${ }^{6}$ ) and inferred new loop formation in RIS cells. e, Differential Hi-C matrix at the IL1 locus (log-fold change TNF $\alpha$ ) at $10 \mathrm{~kb}$ resolution $\mathbf{f}$, Significant differential enhancer-promoter contacts between promoters of differentially expressed genes at the IL1 locus and associated enhancers, aligned with $d$ and e, respectively. 
Figure 3 Correlation between cohesin redistribution and loop rewiring during RIS: a, Number of CTCF and cohesin ChIP-seq peaks with increased (green) and decreased (blue) binding. $\mathbf{b}$, Position of CTCF and cohesin binding changes relative to the growing IMR90 loops $^{6}$; each loop is represented as a radial segment linking the two loop anchors. c,

600 Difference between RIS and growing aggregated $\mathrm{Hi}-\mathrm{C}$ interactions neighbourhoods (20 kb resolution) of IMR90 loops overlapping with significantly decreased cohesin binding at least one loop ends (left). Compare to all IMR90 loops with significantly decreased interactions during RIS (right). d, De novo cohesin (RAD21 and SMC3) binding at the 3' end of IL1B coinciding with the inferred position of the de novo loop formation in RIS, as well as CTCF and H3K27ac THOR-normalized ChIP-seq signal in growing (Grow) and RIS. e, THORnormalized SMC3 ChIP-seq signal at the IL1 locus in RIS via constitutive expression of HRAS $^{\mathrm{G} 12 \mathrm{~V}}$ (pBabe) in IMR90 and WI38 cells, as well as matched growing controls (Vector). f, THOR-normalized ChIP-seq signal of cohesin (RAD21 and SMC3) and CTCF at the IL1 locus in TNF $\alpha$-treated and matched control IMR90 cells. g, Proposed model for the de novo loop

610 formation at the $I L 1$ locus, separating $I L 1 B$ from $I L 1 A$ and $C K A P 2 L$, along with their specific enhancers.

Figure 4 Transcription-dependent cohesin island formation in RIS associated with increased cohesin-cohesin interactions: a, Transcription-dependent cohesin (RAD21 THOR-normalized signal) accumulation at the 3' end of genes in RIS cells, grouped by log-

615 transcripts-per-million (TPM) expression at convergent genes (overlapping extended 3' ends) and isolated genes (no overlap with other genes), with the reference points: middle point between the converging 3' ends and TES, transcriptional end site. In the case of convergent genes, both genes in the pair were in the same expression category. b, THOR-normalized ChIP-seq at the IL1 locus of cohesin (RAD21, SMC3) and CTCF with and without DRB 620 treatment c, Aggregated SMC3 ChIP-seq signal in growing, RIS and RIS with DRB treatment, and CTCF ChIP-seq in RIS over all cohesin islands identified, flanked by $5 \mathrm{~kb}$. d, Difference 
between aggregated interaction neighbourhoods (at $20 \mathrm{~kb}$ resolution) in RIS and in growing of all interactions between cohesin islands and nearby cohesin peaks within $150 \mathrm{~kb}$ of each other (left). The right panel represents significantly increasing interactions during RIS between each cohesin island and nearby cohesin peaks (within $250 \mathrm{~kb}$ either side). e, Cohesin islands at $I L 1 B$ during macrophage terminal differentiation and loop modulation. Reanalysis of $\mathrm{Hi}-\mathrm{C}$ matrices at $5 \mathrm{~kb}$ resolution of THP-1 monocytes and PMA-induced macrophages from Phanstiel et al. ${ }^{15}$ at the IL1 locus, as well as cohesin ChIP-seq (RAD21) from Heinz et al. ${ }^{38}$ in the same cell context.

630 Extended Data Figure 1. Consistency across Hi-C and cHi-C biological replicates: a, HiC-spector agreement scores between each pair of growing and RIS biological replicates between 0 and 1 , indicating poor and good agreement, respectively. b-c, Principal Component Analysis on read counts, which were library size-normalized and filtered for low values, corresponding to interactions at $40 \mathrm{~kb}$ resolution from the growing and $\mathrm{RIS} \mathrm{Hi-C} \mathrm{(b)}$

635 and $\mathrm{cHi}-\mathrm{C}$ (c) libraries. d, Growing and RIS interactions matrix at $20 \mathrm{~kb}$ resolution of the HMGA2 gene and surrounding TADs with matching RNA-seq (TMM-normalized) and ChIPseq tracks of H3K27me3 and H3K27ac, as well as ATAC-seq. e, TADbit three-dimensional modelling of the three TADs marked in d, colouring the HMGA2, IRAK3 and GRIP1 genes as well as the TADs they belong to.

640 Extended Data Figure 2. A/B compartments in growing and RIS: Distribution across each chromosome of the principal component corresponding to $A / B$ compartments (based on correlation with H3K4me1 signal) from PCA performed on growing (G) and RIS Hi-C libraries; positive values (green) mark the A compartment, whereas negative values (blue) mark the B compartment.

645 Extended Data Figure 3. Epigenetic characterization of enhancers and promoters using ATAC-seq and H3K27ac, H3K4me1 and H3K4me3 ChIP-seq signal: a, Enhancers defined 
by H3K27ac peaks which also have ATAC-seq and H3K4me1 and low H3K4me3 ChIP-seq signal, split by regions common between growing $(\mathrm{G})$ and RIS and specific to each condition. b, Promoters defined as $5 \mathrm{~kb}$ regions around the TSS of every protein-coding gene, showing low H3K4me1 and high H3K4me3 signal.

\section{Extended Data Figure 4. Enhancer-Promoter (EP) Network from capture Hi-C: a,} Differential EP interactions network based on annotated cHi-C significant interaction changes at HindIII resolution. Two boxed components are also shown in Fig. 2a. b, comparison between EP interactions annotated from genome-wide $\mathrm{Hi}-\mathrm{C}$ analysis at resolutions between

$65510 \mathrm{~kb}$ and $100 \mathrm{~kb}$, and EP interactions annotated from $\mathrm{cHi}-\mathrm{C}$ (represented in a) filtered with different strategies such as larger enhancers or FDR threshold of the gene; left - percentage of EP annotated from $\mathrm{Hi}-\mathrm{C}$ but not from $\mathrm{cHi}-\mathrm{C}$ ('false positives') in the captured regions, and right - percentage of EP annotated from $\mathrm{Hi}-\mathrm{C}$ as well as from $\mathrm{cHi}-\mathrm{C}$ ('true positives'); the filtering strategy highlighted (enhancers larger than $7.5 \mathrm{~kb}$ ) and bin sizes smaller than $30 \mathrm{~kb}$ 660 minimise the EP annotated from $\mathrm{Hi}-\mathrm{C}$ but not from $\mathrm{cHi}-\mathrm{C}$ and maximise the ones annotated.

Extended Data Figure 5. Genome-wide Hi-C EP differential network: a, EP network using the filtering strategy based on EP changes annotated from cHi-C (Extended Data Fig. 4b, enhancers larger than $7.5 \mathrm{~kb}$ and resolution higher than $30 \mathrm{~kb}$, i.e. bin sizes smaller than 30 $\mathrm{kb}$ ) on each chromosome, with the vertical axis representing enhancers and the right and the

665 left axes corresponding to up-regulated and down-regulated genes, respectively. b, Gene enrichment with EnrichR against the WikiPathways 2019 database of up-regulated (left) and down-regulated (right) genes in the genome-wide Hi-C EP differential network; the dotted grey line corresponds to 0.05 adjusted p-value, the selected threshold for significant enrichment. Terms were manually simplified and obvious redundancy was removed.

670 Extended Data Figure 6. Down-regulation of CCNA2 associated with decreased EP interactions: Growing and RIS Hi-C matrices (10 kb resolution) centred on the IMR90 loop ${ }^{6}$ 
consisting of the CCNA2 gene promoter and associated enhancers, as well as $\mathrm{cHi}-\mathrm{C}$ differential log-fold change matrix (5 $\mathrm{kb}$ resolution) of this loop and significant decreased interactions (blue arcs) between the CCNA2 gene promoter and associated enhancers.

675 Extended Data Figure 7. RAD21 binding gains and losses in growing and RIS: Profiles and heatmaps of THOR-normalized RAD21 ChIP-seq signal in growing and RIS, centred on peaks overlapping regions with significantly increased or decreased binding, as determined with THOR at FDR 0.05.

\section{Extended Data Figure 8: Interaction changes during RIS at the MMP locus suggesting}

680 increased spatial separation around MMP1: a, cHi-C differential interaction matrix (HindIII resolution) at the MMP locus, consisting of the promoters of $M M P 10, M M P 1, M M P 3, M M P 12$ and associated enhancers, as well as the significant EP contacts between them (green and blue arcs, corresponding to increased and decreased interactions, respectively). b, ChIP-seq THOR-normalized tracks at the MMP locus marked in a, with dotted lines of RAD21, SMC3,

685 CTCF and H3K27ac in growing and RIS, as well as the MMP genes. c, SMC3 CHIP-seq of the MMP locus in RIS IMR90 and WI38 cells via constitutive expression of oncogenic HRASG12V and matched growing controls (empty vector). d, RAD21, SMC3 and CTCF ChIP-seq in RIS with and without DRB treatment (transcription elongation inhibitor) at the MMP locus with cohesin islands at the end of MMP1 and MMP3.

690 Extended Data Figure 9: Cohesin islands in RIS IMR90 cells and THP-1 macrophages: a, Distribution of SMC3 ChIP-seq signal between the 3' ends of convergent gene pairs, where both genes are in one of the four expression categories defined based on their logtranscripts-per-million (TPM* signifies TPM of both genes in the pair) in growing and RIS, respectively. b, SMC3 signal around 3' end (TES) of isolated genes averaged by four expression groups defined by log-TPM of the genes in growing and RIS, respectively. c, RAD21 and SMC3 ChIP-seq signal at the 3' end of 559 genes which are highly expressed in 
growing and RIS but more highly in growing. d, Gene enrichment pathways of genes with cohesin islands against WikiPathways 2019 (dotted line corresponds to the significance threshold, 0.05 p-adjusted). e, SMC3 ChIP-seq signal of constitutive HRAS-G12V-induced senescence and matched controls (empty vector), in IMR90 and WI38 cells, over the cohesin islands identified from RIS compared with RIS with DRB treatment. f, SMC3 ChIP-seq signal of RIS, TNF $\alpha$-treated and matched control IMR90 cells over the cohesin islands. g, RAD21 ChIP-seq by Heinz et al. ${ }^{38}$ around the 3' end of genes grouped by expression levels (RNAseq from Phanstiel et al. $^{15}$ ) in THP-1 monocytes and PMA-induced macrophages. $\mathbf{h}$, Monocyte and macrophage RAD21 ChIP-seq by Heinz et al. ${ }^{38}$ over the cohesin islands defined in RIS IMR90 cells.

Extended Data Table 1: List of captured regions with hg19 coordinates

Extended Data Table 2: Number of valid reads aligned from each $\mathrm{Hi}-\mathrm{C}$ and $\mathrm{cHi}-\mathrm{C}$ library, as well as the number of reads corresponding to various types of artefacts

710 Extended Data Table 3: Genes up-regulated during RIS which also show reduced interactions with H3K27me3 regions, escaping heterochromatic three-dimensional neighbourhoods

Extended Data Table 4: Enhancer-promoter differential contacts from cHi-C (HindIII resolution) and $\mathrm{Hi}-\mathrm{C}$ (at different resolutions)

715 Extended Data Table 5: Genes with cohesin at their 3'ends during RIS

39. Schmidt, D. et al. ChIP-seq: Using high-throughput sequencing to discover proteinDNA interactions. Methods 48, 240-248 (2009).

40. Sakata, T., Shirahige, K. \& Sutani, T. ChIP-seq Analysis of Condensin Complex in 
271 (Humana Press, New York, NY, 2017).

41. Kimura, H., Hayashi-Takanaka, Y., Goto, Y., Takizawa, N. \& Nozaki, N. The Organization of Histone H3 Modifications as Revealed by a Panel of Specific Monoclonal Antibodies. Cell Struct. Funct. 33, 61-73 (2008).

42. Minamino, M. et al. Esco1 Acetylates Cohesin via a Mechanism Different from That of Esco2. Curr. Biol. 25, 1694-1706 (2015).

43. Schoenfelder, S., Javierre, B.-M., Furlan-Magaril, M., Wingett, S. W. \& Fraser, P. Promoter Capture Hi-C: High-resolution, Genome-wide Profiling of Promoter Interactions. J. Vis. Exp. (2018). doi:10.3791/57320

44. Nagano, T. et al. Comparison of Hi-C results using in-solution versus in-nucleus ligation. Genome Biol. 16, 175 (2015).

45. Servant, N. et al. HiC-Pro: an optimized and flexible pipeline for Hi-C data processing. Genome Biol. 16, 259 (2015).

46. Li, H. et al. The Sequence Alignment/Map format and SAMtools. Bioinformatics 25, 2078-2079 (2009).

47. Lieberman-Aiden, E. et al. Comprehensive mapping of long-range interactions reveals folding principles of the human genome. Science 326, 289-93 (2009).

48. Serra, F. et al. Automatic analysis and 3D-modelling of Hi-C data using TADbit reveals structural features of the fly chromatin colors. PLOS Comput. Biol. 13, e1005665 (2017).

49. Russel, D. et al. Putting the Pieces Together: Integrative Modeling Platform Software for Structure Determination of Macromolecular Assemblies. PLoS Biol. 10, e1001244 (2012).

50. Langmead, B. \& Salzberg, S. L. Fast gapped-read alignment with Bowtie 2. Nat. Methods 9, 357-359 (2012).

51. Amemiya, H. M., Kundaje, A. \& Boyle, A. P. The ENCODE Blacklist: Identification of 
Problematic Regions of the Genome. Sci. Rep. 9, 9354 (2019).

52. Consortium, T. E. P. An integrated encyclopedia of DNA elements in the human genome. Nature 489, 57-74 (2012).

53. Feng, J., Liu, T., Qin, B., Zhang, Y. \& Liu, X. S. Identifying ChIP-seq enrichment using MACS. Nat. Protoc. 7, 1728-1740 (2012).

54. Carroll, T. S., Liang, Z., Salama, R., Stark, R. \& de Santiago, I. Impact of artifact removal on ChIP quality metrics in ChIP-seq and ChIP-exo data. Front. Genet. 5, 75 (2014).

55. Allhoff, M., Seré, K., F. Pires, J., Zenke, M. \& G. Costa, I. Differential peak calling of ChIP-seq signals with replicates with THOR. Nucleic Acids Res. 44, e153 (2016).

56. Ramírez, F. et al. deepTools2: a next generation web server for deep-sequencing data analysis. Nucleic Acids Res. 44, W160-W165 (2016).

57. Dobin, A. et al. STAR: ultrafast universal RNA-seq aligner. Bioinformatics 29, 15-21 (2013).

58. Liao, Y., Smyth, G. K. \& Shi, W. featureCounts: an efficient general purpose program for assigning sequence reads to genomic features. Bioinformatics 30, 923-930 (2014).

59. McCarthy, D. J., Chen, Y. \& Smyth, G. K. Differential expression analysis of multifactor RNA-Seq experiments with respect to biological variation. Nucleic Acids Res. 40, 4288-4297 (2012).

60. Chen, E. Y. et al. Enrichr: interactive and collaborative HTML5 gene list enrichment analysis tool. BMC Bioinformatics 14, 128 (2013).

61. Kuleshov, M. V. et al. Enrichr: a comprehensive gene set enrichment analysis web server 2016 update. Nucleic Acids Res. 44, W90-W97 (2016).

62. Imakaev, M. et al. Iterative correction of $\mathrm{Hi}-\mathrm{C}$ data reveals hallmarks of chromosome organization. Nat. Methods 9, 999-1003 (2012). 
63. Robinson, J. T. et al. Integrative genomics viewer. Nat. Biotechnol. 29, 24-6 (2011).

64. Chandra, T. et al. Independence of Repressive Histone Marks and Chromatin Compaction during Senescent Heterochromatic Layer Formation. Mol. Cell 47, 203214 (2012).

65. Parry, A. J. et al. NOTCH-mediated non-cell autonomous regulation of chromatin structure during senescence. Nat. Commun. 9, 1840 (2018). 


\section{Fig. 1_Olan et al.}

a

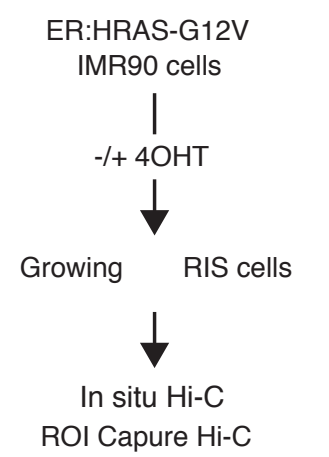

b

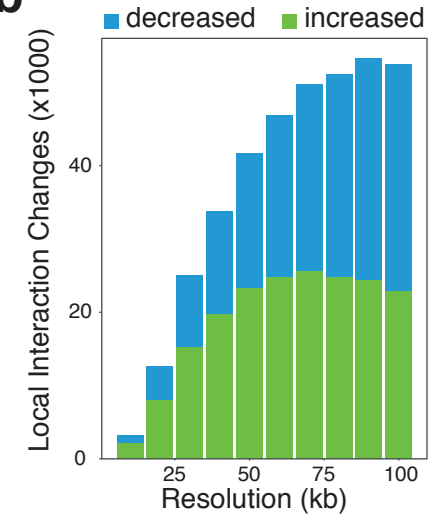

d
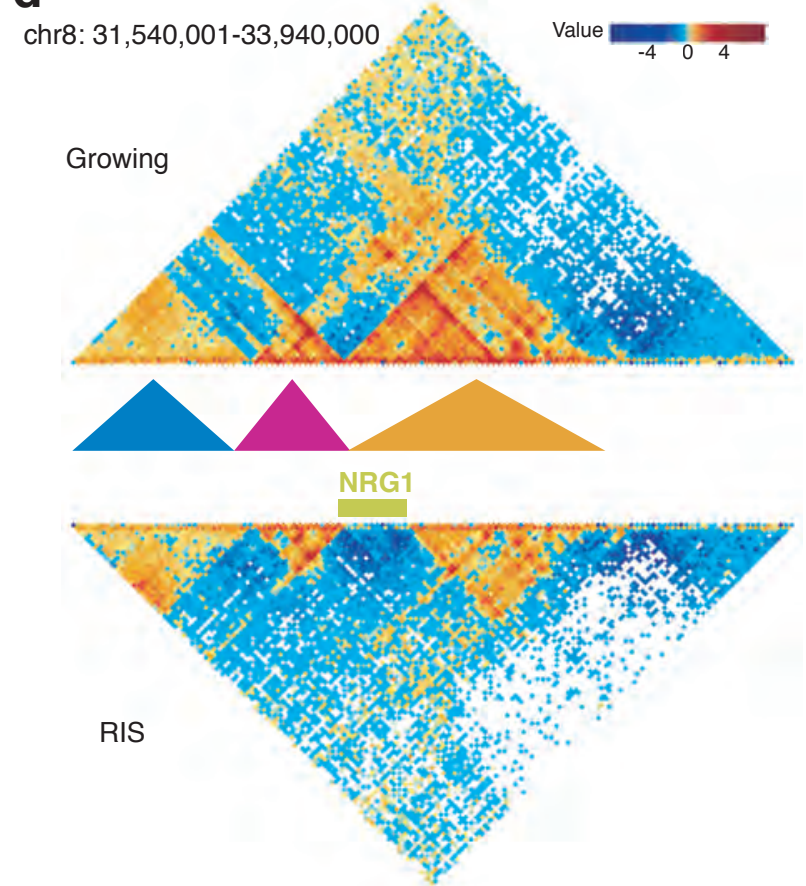

Grow
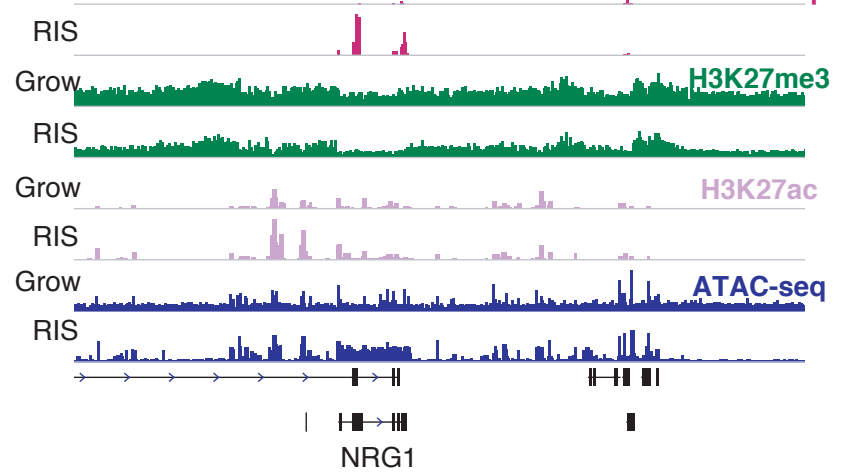

C

C chr8: $1-146,364,022$

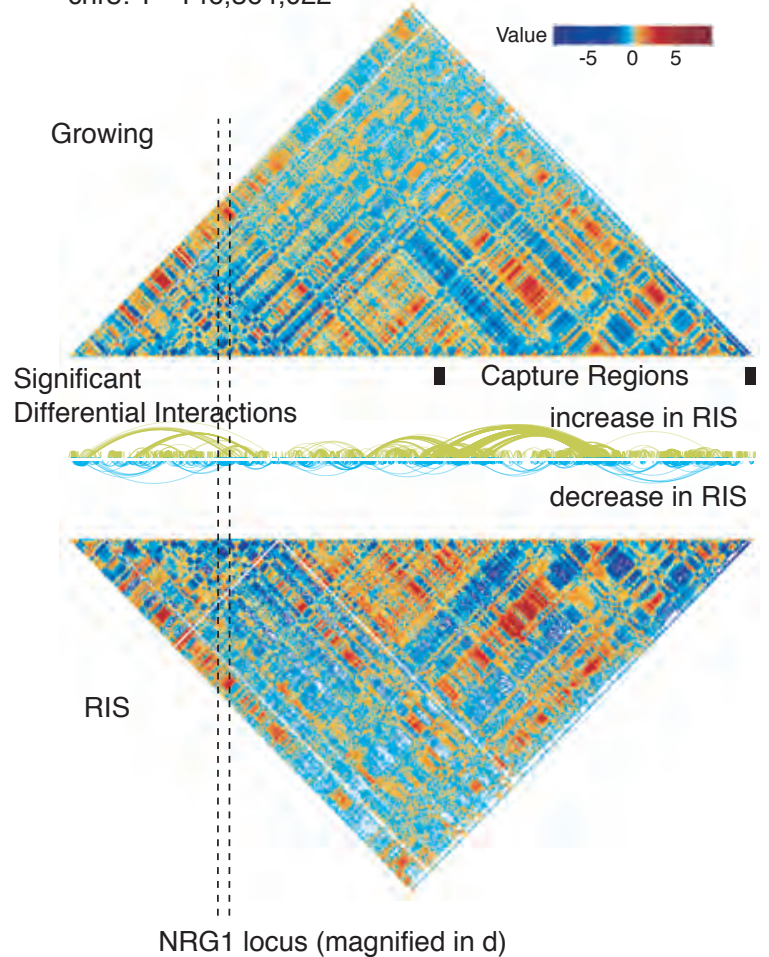

e

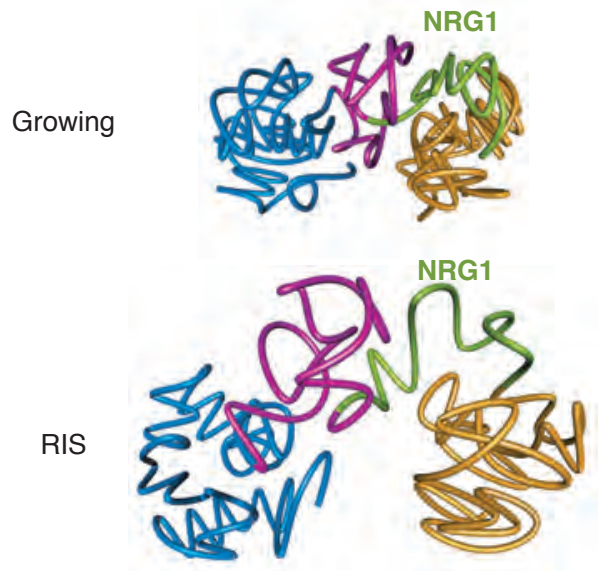

Figure 1 Changes in chromatin interactions identified with Hi-C during RIS: a, Experimental setup of control Growing and RAS-induced senescent (RIS) IMR90 cells. ROI, regions of interest. b, Number of significant interaction changes during RIS at resolutions between 10 and $100 \mathrm{~kb}$. c, Hi-C matrices (300 kb resolution) of chromosome 8 in Growing and RIS cells; arcs represent significant interaction changes (100 kb resolution); black boxes represent captured regions. $\mathrm{d}$, Hi-C interaction matrices at the NRG1 locus, marked by dotted lines in c at $20 \mathrm{~kb}$ resolution, with matching tracks for RNA-seq, ChIP-seq, and ATAC-seq; coloured triangles represent TADs. e, Three-dimensional interaction modelling with TADbit at the NRG1 locus in Growing and RIS, including NRG1 (green) and surrounding TADs marked in $\mathbf{d}$. 
a

\section{Fig. 2_Olan et al.}

\section{b}

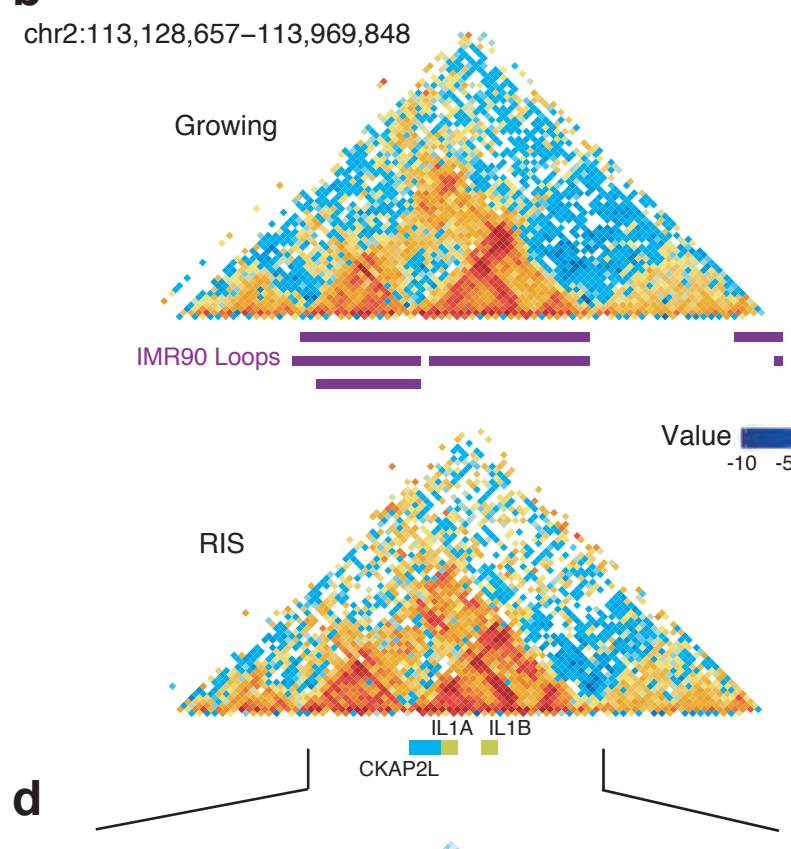

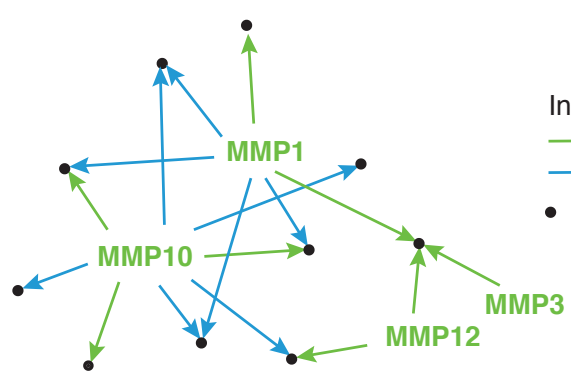

C

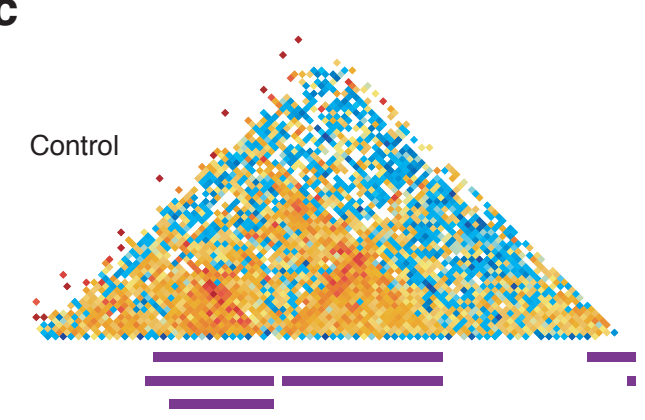

chr2:113,371,909-113,727,090

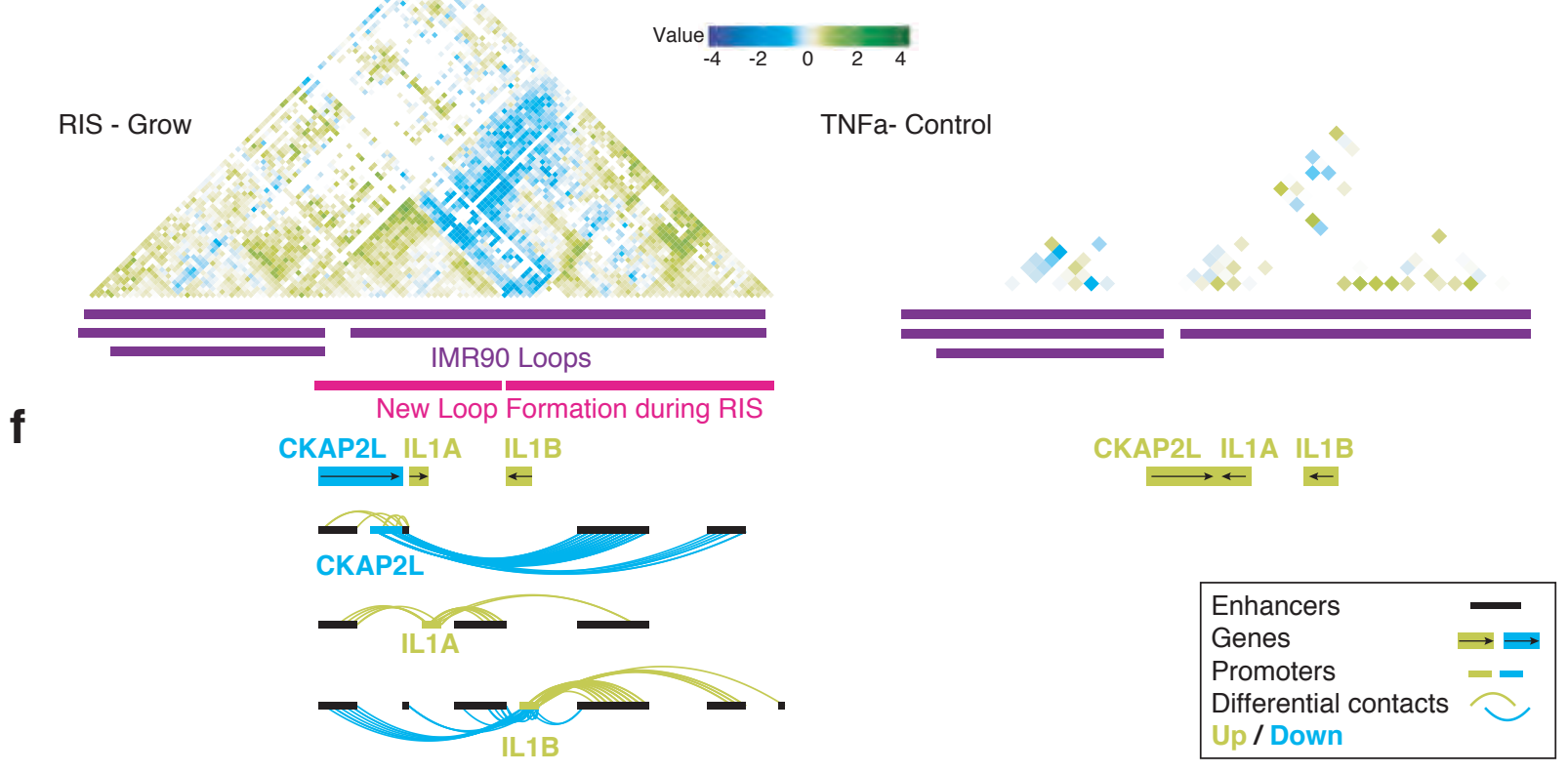

Figure 2 Reorganization of the local chromatin neighbourhood at the IL1 locus: a, Differential enhancer-promoter contacts involving IL1 and MMP in cHi-C. b-c, Hi-C matrices (10 kb resolution) of the IL 1 locus corresponding to growing and RIS IMR90 cells (b) and control and TNFa-treated IMR90 cells from Jin et al. (c). d, Differential capture Hi-C matrix at the IL1 locus (log-fold change of RIS/growing interactions) at HindIII resolution, with annotated growing IMR90 loops (from Rao et al.) and inferred new loop formation in RIS cells. e, Differential Hi-C matrix at the IL1 locus (log-fold change TNFa/control) at $10 \mathrm{~kb}$ resolution $\mathbf{f}$, Significant differential enhancer-promoter contacts between promoters of differentially expressed genes at the IL 1 locus and associated enhancers, aligned with $\mathbf{d}$ and $\mathbf{e}$, respectively. 
a

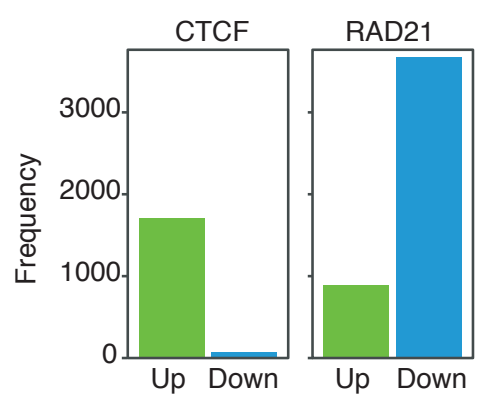

b
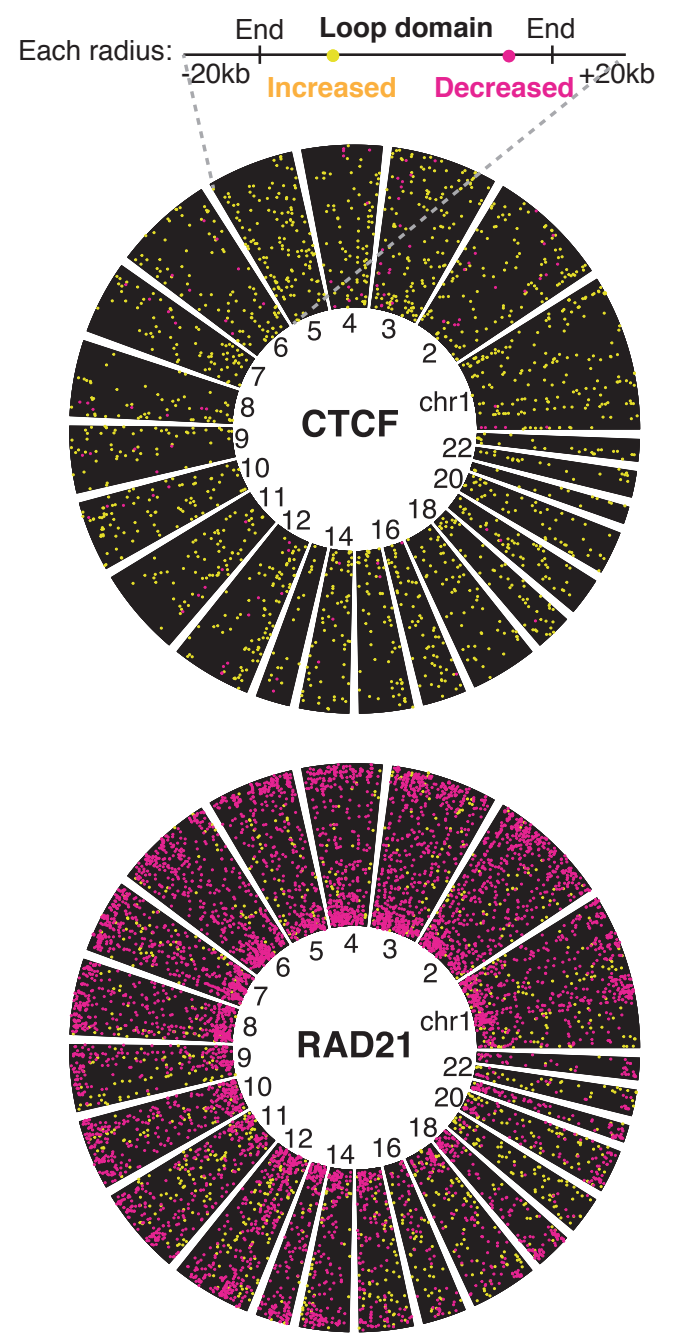

C

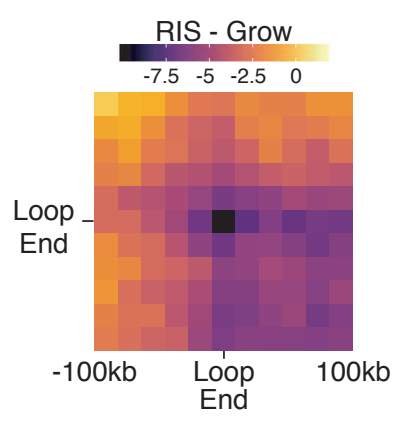

Fig. 3_Olan et al.

d

chr2:113,371,909-113,727,090

IMR90 Loops

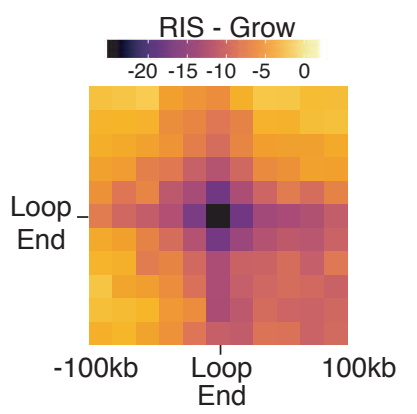

New RIS loops

RAD21

Grow

SMC3

RIS

Grow

RIS

CTCF

Grow RIS |

H3K27ac

e

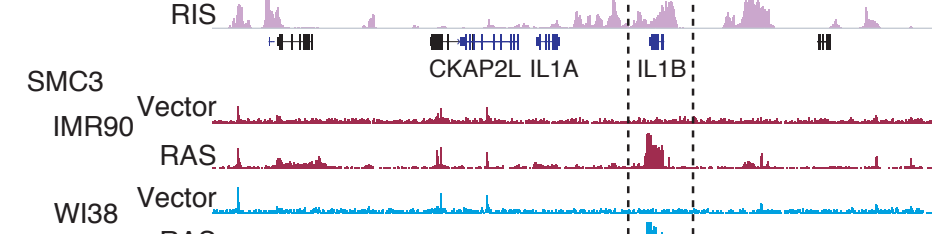

$\mathbf{f}$

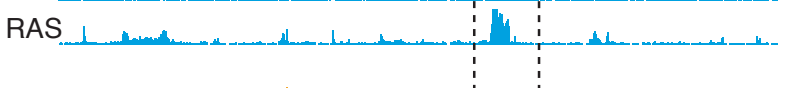

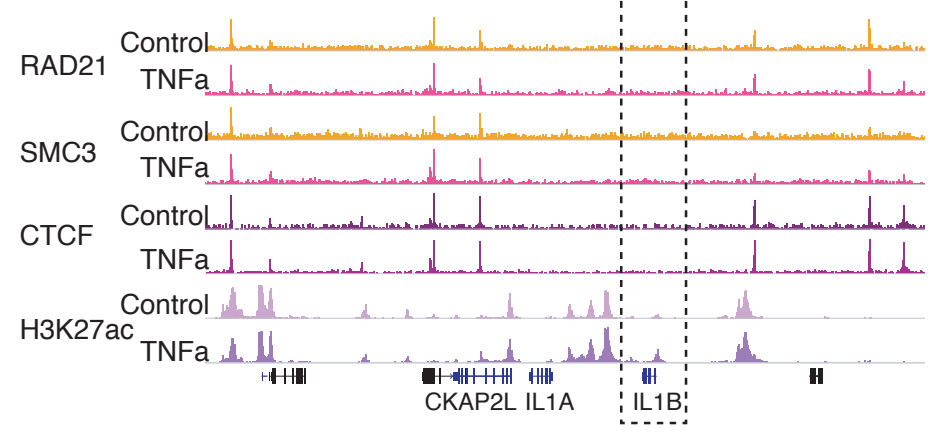

9
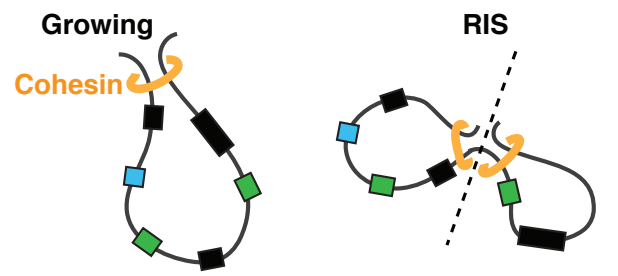

Figure 3 Correlation between cohesin retribution and loop rewiring during RIS: a, Number of CTCF and cohesin ChIP-seq peaks with increased (green) and decreased (blue) binding. $\mathbf{b}$, Position of CTCF and cohesin binding changes relative to the growing IMR90 loops; each loop is represented as a radial segment linking the two loop anchors. c, Difference between RIS and growing aggregated Hi-C interactions neighbourhoods (20 kb resolution) of IMR90 loops overlapping with significantly decreased cohesin binding at least one loop ends (left). Compare to all IMR90 loops with significantly decreased interactions during RIS (right). d, De novo cohesin (RAD21 and SMC3) binding at the 3' end of IL1B coinciding with the inferred position of the de novo loop formation in RIS, as well as CTCF and H3K27ac THOR-normalized ChIP-seq signal in growing (Grow) and RIS. e, THOR-normalized SMC3 ChIP-seq signal at the IL1 locus in RIS via constitutive expression of HRASG12V (pBabe) in IMR90 and WI38 cells, as well as matched growing controls (Vector). f, THOR-normalized ChIP-seq signal of cohesin (RAD21 and SMC3) and CTCF at the IL 1 locus in TNFa-treated and matched control IMR90 cells. g, Proposed model for the de novo loop formation at the IL1 locus, separating IL1B from IL1A and CKAP2L, along with their specific enhancers. 
a

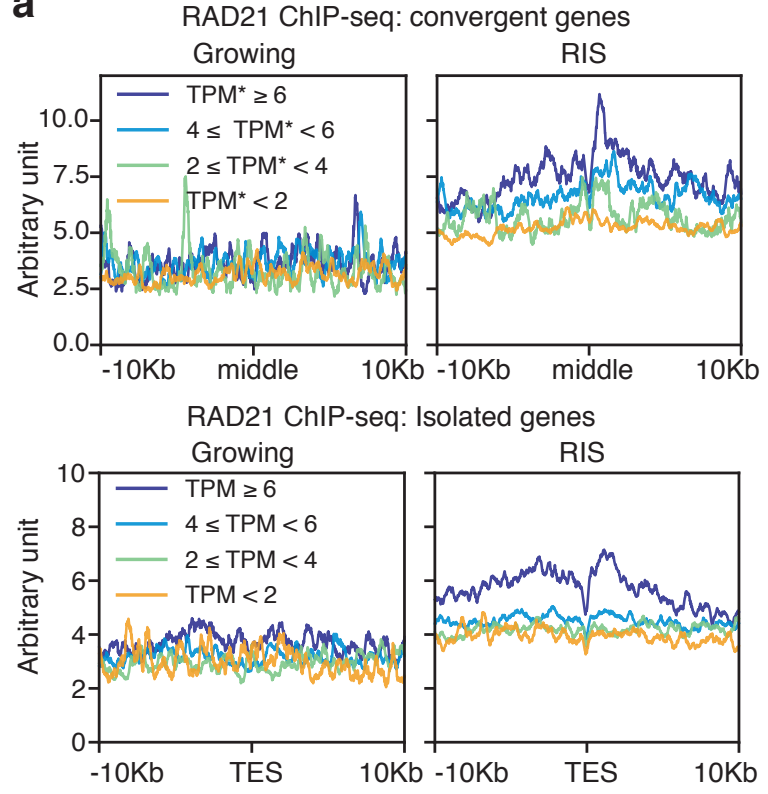

C

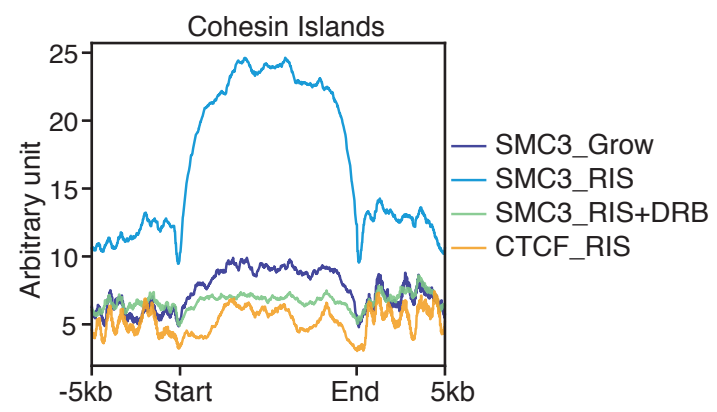

d

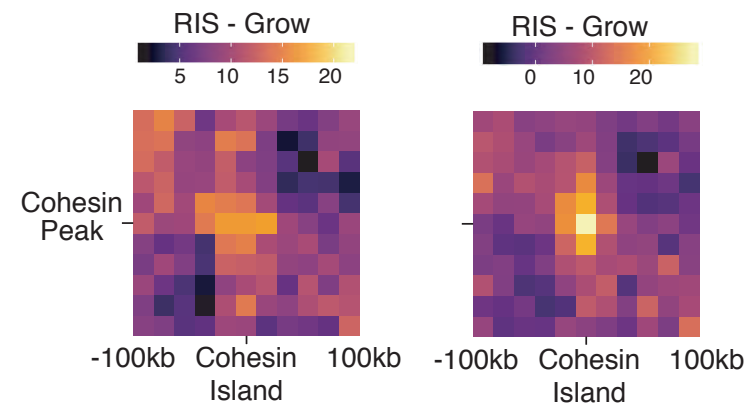

\section{Fig. 4_Olan et al.}

b chr2:113,371,909-113,727,090

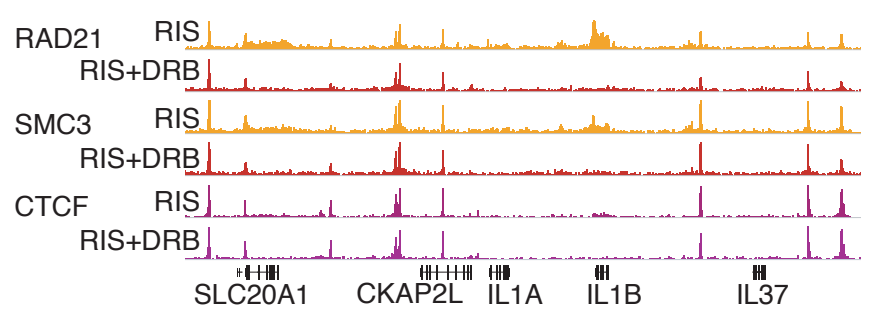

e

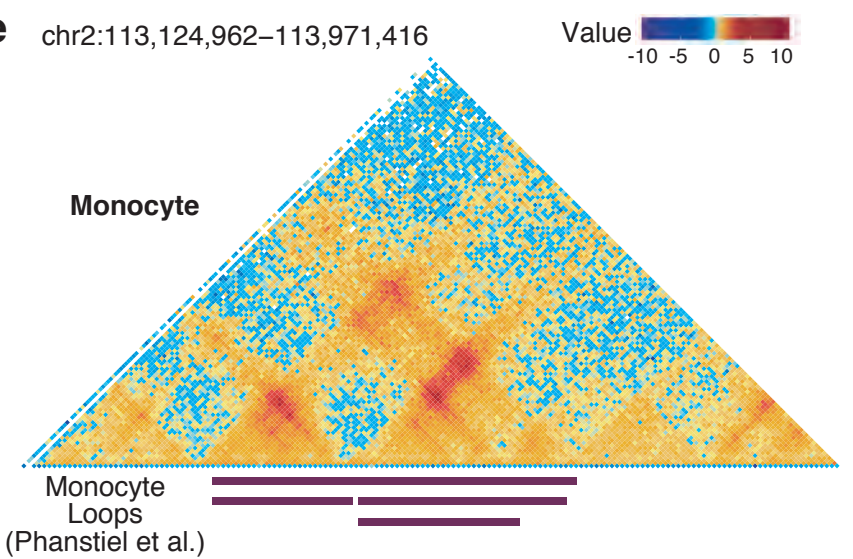

(Phanstiel et al.)

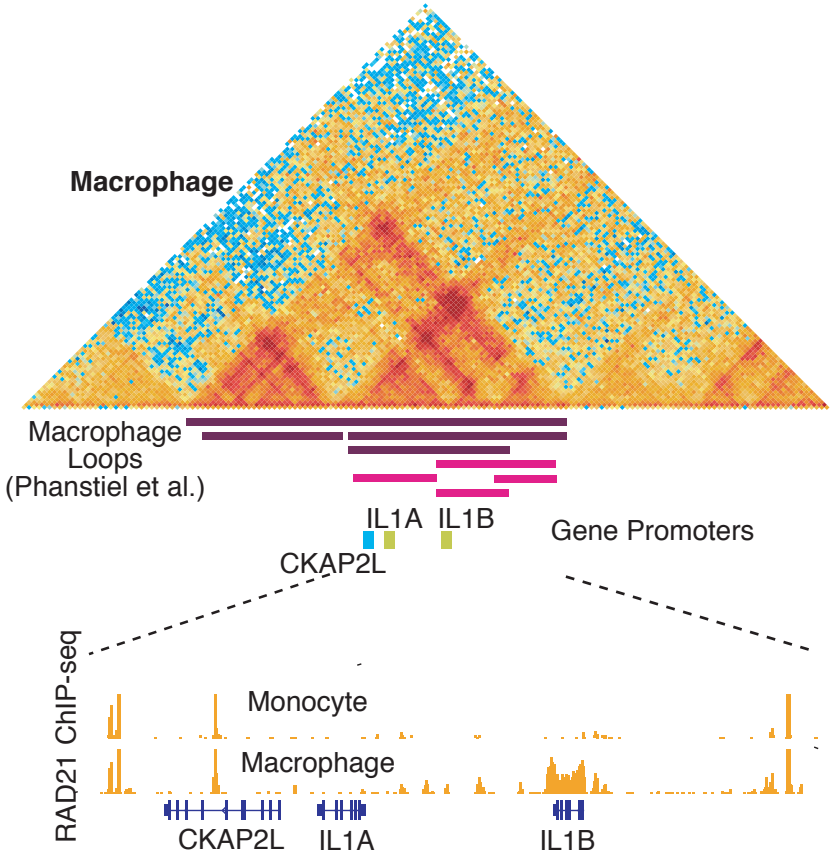

Figure 4 Transcription-dependent cohesin island formation in RIS associated with increased cohesin-cohesin interactions: a, Transcription-dependent cohesin (RAD21 THOR-normalized signal) accumulation at the 3' end of genes in RIS cells, grouped by log-transcripts-per-million (TPM) expression at convergent genes (overlapping extended 3' ends) and isolated genes (no overlap with other genes), with the reference points: middle point between the converging 3' ends and TES, transcriptional end site. In the case of convergent genes, both genes in the pair were in the same expression category. b, THOR-normalized ChIP-seq at the IL1 locus of cohesin (RAD21, SMC3) and CTCF with and without DRB treatment c, Aggregated SMC3 ChIP-seq signal in growing, RIS and RIS with DRB treatment, and CTCF ChIP-seq in RIS over all cohesin islands identified, flanked by $5 \mathrm{~kb}$. $\mathbf{d}$, Difference between aggregated interaction neighbourhoods (at $20 \mathrm{~kb}$ resolution) in RIS and in growing of all interactions between cohesin islands and nearby cohesin peaks within $150 \mathrm{~kb}$ of each other (left). The right panel represents significantly increasing interactions during RIS between each cohesin island and nearby cohesin peaks (within $250 \mathrm{~kb}$ either side). e, Cohesin islands at IL1B during macrophage terminal differentiation and loop modulation. Reanalysis of Hi-C matrices at $5 \mathrm{~kb}$ resolution of THP-1 monocytes and PMA-induced macrophages from Phanstiel et al. at the IL1 locus, as well as cohesin ChIP-seq (RAD21) from Heinz et al. in the same cell context. 